Check for updates

Cite this: RSC Adv., 2017, 7, 18358

Received 14th January 2017

Accepted 13th March 2017

DOI: 10.1039/c7ra00570a

rsc.li/rsc-advances

\section{Comparatively studying the local atomic structures of metallic glasses upon cyclic-loading by computer simulations $\dagger$}

\author{
M. H. Yang, (D) J. H. Li* and B. X. Liu \\ Based on a newly constructed Ni-Zr potential, Molecular dynamics and Monte Carlo simulations predict the \\ amorphization driving force for each alloy and pinpoint the optimized value of 40 at\% Zr. Through a variety \\ of local structural analysis methods, it is revealed that $\mathrm{Ni}_{x} \mathrm{Zr}_{100-x}$ MGs exhibit a combination of icosahedral-, \\ fcc- and hcp-like configurations, while the icosahedra or distorted icosahedra cover a dominant fraction. \\ Interestingly, the lower-CN clusters, such as $\langle 0,2,8,1\rangle$, may play a significant role in atomic mobility, \\ while the icosahedron is less sensitive to the faster mobility.
}

\section{Introduction}

Understanding the relationship between local atomic structures and mechanical behaviors in metallic glasses (MGs) is of vital importance for current applications and emerging technologies. ${ }^{1-6}$ There is increasing evidence to suggest that the deformation behavior of MGs is driven by the cooperative organization of irreversible rearrangements of small dynamical clusters, which are triggered by the nonlocal redistribution of elastic stress. ${ }^{7-9}$ The sequence of such plastic events can contribute to an avalanche process characterized by a power-law scaling of the average stress or energy drop with system size. ${ }^{\mathbf{1 0 , 1 1}}$ In addition, the local atomic-level structure of MGs varies from site to site due to their intrinsic structure. The spatial connectivity and various kinds of local structural motifs in MGs significantly influence the mechanical response of the macroscopic sample under loading. In this case, the structural heterogeneity necessarily contributes to the mechanical heterogeneity, and this structural-mechanical heterogeneity still remains a mystery and needs further insightful research.

In practical applications, service conditions that introduce cyclic variations in stresses are inevitably involved, and the resistance of MGs to the onset and progression of localized deformation under cyclic loading has attracted considerable scientific and technological interests. ${ }^{12,13}$ Due to the small length scales and short time scales, the atomic-scale processes underlying shear localization under cyclic deformation are difficult to study experimentally. Meanwhile, atomistic simulations, particularly Molecular dynamics (MD),

Key Laboratory of Advanced Materials (MOE), School of Materials Science and Engineering, Tsinghua University, Beijing 100084, China. E-mail: lijiahao@mail. tsinghua.edu.cn

$\dagger$ Electronic supplementary information (ESI) available. See DOI: 10.1039/c7ra00570a are playing a significant role in elucidating the structure of MGs and monitoring the evolution of the atomic structure during mechanical deformation. It is well accepted that icosahedra or distorted icosahedra are important local structural motifs for the dynamical slowdown in metallic supercooled liquids, stability of supercooled liquids against crystallization, and formation of metallic glasses. ${ }^{\mathbf{1 4}}$ For example, the enhanced full icosahedral ordering with increasing $\mathrm{Cu}$ content in $\mathrm{Cu}-\mathrm{Zr}$ models matches hand in hand with the appropriate composition ratios and bond types, and these chemical, topological and kinetic structural effects lead to the higher resistance to shear transformations and hence also reduce the propensity for plasticity. ${ }^{15}$ Although $\mathrm{Ni}$ and $\mathrm{Cu}$ are neighbors in the periodic table and have many similarities, there is so far little or no atomic-level understanding of the structural-mechanical properties in Ni-Zr MGs under cyclic deformation.

In the present work, Molecular dynamics (MD) and Monte Carlo (MC) simulations are applied to study the local atomic structure characteristics, and their effect on the mechanical properties of the Ni-Zr MGs was further explored. The paper is organized as follows: based on the newly proposed potential, the local atomic structure of Ni-Zr binary alloys was quantitatively determined in terms of the pair-correlation functions, static structure factor $S(q),{ }^{\mathbf{1 6}}$ Honeycutt-Andersen pair analysis, ${ }^{17}$ coordination number, Voronoi tessellation method ${ }^{\mathbf{1 8}}$ and cluster alignment method. ${ }^{19}$ In addition, the recently proposed molecular dynamics simulation of dynamic mechanical spectroscopy (MD-DMS) was used to analyze the internal friction (IF) characteristics, which are responsible for the transport and damping properties of amorphous materials. We attempt to provide an explicit picture of the local atomic structures in $\mathrm{Ni}-\mathrm{Zr} \mathrm{MGs}$ and to further clarify the structure-property relationship. 


\section{Method and model}

\subsection{Molecular dynamics (MD) and Monte Carlo (MC) simulations}

In practice, the process of producing metallic glass always occurs under non-equilibrium conditions, and the related kinetic conditions are extremely restricted. As a result, the phase competing against the amorphous phase is the solid solution with one of the three simple structures, i.e. fcc, hcp, or bcc. Recent studies have revealed that there exists no pronounced differences in the local atomic structure and mechanical behaviour between solid-state amorphization and liquid melt quenching (LMQ) production methods. ${ }^{20}$ Applying the newly constructed long-range empirical $\mathrm{Ni}-$ $\mathrm{Zr}$ potential, ${ }^{21}$ a sequence of MD simulations was implemented using Large-scale Atomic/Molecular Massively Parallel Simulator (LAMMPS) packages to study the relative stabilities of $\mathrm{Ni}-\mathrm{Zr}$ solid solutions versus their amorphous counterparts.

$\mathrm{Ni}_{50} \mathrm{Zr}_{50}$ and $\mathrm{Ni}_{65} \mathrm{Zr}_{35}$ belong to a fcc solid solution model with dimensions of $\sim 60.0 \times 60.0 \times 60.0 \AA^{3}$, while $\mathrm{Ni}_{35} \mathrm{Zr}_{65}$ belongs to a hep solid solution model with dimensions of $\sim 45.3$ $\times 78.4 \times 73.9 \AA^{3}$. Both the fcc and hcp solid solution models consist of $15 \times 15 \times 15 \times 4=13500$ atoms. Periodic boundary conditions were adopted in three Cartesian directions. With a time step of 5 femtoseconds, MD simulation was performed in the framework of an isothermal-isobaric ensemble, and conducted at $300 \mathrm{~K}$ and $0 \mathrm{~Pa}$ for 1 million time steps to be fully relaxed. As shown in the ESI, $\uparrow$ the glass transition temperature $T_{\mathrm{g}}$ of the $\mathrm{Ni}_{35} \mathrm{Zr}_{65}, \mathrm{Ni}_{50} \mathrm{Zr}_{50}$ and $\mathrm{Ni}_{65} \mathrm{Zr}_{35}$ alloys are about $1000 \mathrm{~K}$, $1100 \mathrm{~K}$ and $1200 \mathrm{~K}$, respectively. Meanwhile, a series of Monte Carlo (MC) simulations were implemented to calculate the formation energy of the solid solutions. MC simulations were performed at $300 \mathrm{~K}$ and $0 \mathrm{~Pa}$ in the isothermal-isobaric ensemble. ${ }^{22}$

\subsection{Simulation of dynamic mechanical spectroscopy}

To simulate the real case of dynamic mechanical spectroscopy experiments, a time-period sinusoidal strain $\varepsilon(t)=\varepsilon_{\mathrm{A}} \sin \left(2 \pi f_{\omega} t\right)$ was applied in the $x$ direction of the obtained MGs, where $f_{\omega}$ is the frequency of the oscillation period and is selected as 1,10 and $100 \mathrm{GHz}$ in the present work. We fixed the strain rate amplitude $\varepsilon_{\mathrm{A}}$ as $2.0 \%$ in the linear elastic deformation stage. For every MD-DMS, 20 full period cycles were used and thus $t$ was in the range of $0,20 / f_{\omega}$. The resultant stress was fitted as $\sigma(t)=\sigma_{0}+$ $\sigma_{\mathrm{A}} \sin \left(2 \pi f_{\omega} t+\delta\right)$, where $\sigma_{0}$ is a constant term and usually small, and $\delta$ is the phase difference between stress and strain. The storage $\left(E^{\prime}\right)$ and loss $\left(E^{\prime \prime}\right)$ modulus are calculated and expressed by

$$
\begin{aligned}
& E^{\prime}=\sigma_{\mathrm{A}} / \varepsilon_{\mathrm{A}} \cos (\delta), \\
& E^{\prime \prime}=\sigma_{\mathrm{A}} / \varepsilon_{\mathrm{A}} \sin (\delta) .
\end{aligned}
$$

At a given temperature, the MD-DMS was performed under a constant number, volume and temperature (NVT) ensemble during the cyclic deformations. ${ }^{23,24}$

\subsection{Cluster alignment method}

A recently developed order-mining scheme, i.e. an atomic cluster alignment method, has been adopted to identify and characterize not only the short-range but also medium-range structural order in metallic liquids and glasses. ${ }^{25-27}$ In this method, one cluster is fixed as a template and aligned with other clusters by rigid translation and rotation to minimize the overall mean square distances between the atoms in different clusters. In the present work, a cluster around the Ni atoms is extracted from the snapshot and aligned against an ideal bcc, fcc, hcp and ICO template. In order to describe the structural similarity between the template and the aligned cluster, an alignment score is defined as ${ }^{19}$

$$
f=\min _{0.80 \leq \alpha \leq 1.2}\left(\frac{1}{N} \sum_{i=1}^{N} \frac{\left(\vec{r}_{i \mathrm{c}}-\alpha \vec{r}_{i t}\right)^{2}}{\left(\alpha \vec{r}_{i t}\right)^{2}}\right)^{1 / 2},
$$

where $\vec{r}_{i \mathrm{c}}$ and $\vec{r}_{i \mathrm{t}}$ are the atom positions in the aligned cluster and template, respectively and $\alpha$ is a coefficient to allow an appropriate scaling for the bond length in the templates. A smaller score indicates a higher similarity between the two independent cluster motifs. In order to achieve the global optimum alignment configuration, we adopted 2000 alignment runs with different random initial cluster rotations and obtained the minimum fitting score to measure the structural similarity.

\section{Results and discussion}

\subsection{Metallic glass formation of Ni-Zr alloys}

The concept of glass-forming ability (GFA) is frequently related to the ease or difficulty of the ability to form glass, and this term can be understood in two distinct ways. Firstly, the glassforming ability can be considered as the frustration of crystallization during freezing of the liquid melt for a specific alloy. In practice, a few simple criteria have been proposed, such as the critical dosage $D_{\mathrm{c}}{ }^{29}$ the supercooled liquid region $\Delta T_{\mathrm{xg}}\left(=T_{\mathrm{x}}-\right.$ $\left.T_{\mathrm{g}}\right)^{30}$ and the new parameter $\gamma=T_{\mathrm{x}} /\left(T_{\mathrm{g}}+T_{\mathrm{m}}\right),{ }^{31}$ to describe the GFA of an alloy. Secondly, the glass-forming ability can be depicted as a competition between the crystalline and amorphous phases as an alloy composition function for a specific alloy system. In this case, the glass-forming range (GFR) is employed to indicate the composition range within which the amorphous phase is energetically favored to form..$^{32}$ Therefore, the GFA of an alloy system is quantitatively related to its GFR, i.e. the wider the GFR, the greater the GFA of an alloy system.

According to the simulation results of a Ni-Zr-Mo system, ${ }^{33}$ the GFA/GFR of the Ni-Zr system is determined to be within 2080 at $\% \mathrm{Zr}$ as shown in Fig. 1, matching well with the mechanical alloying experimental results of $17-76$ at $\% \mathrm{Zr}^{34}{ }^{34}$ In addition, we define the $E_{\text {am }}$ as the energy per atom of the $\mathrm{Ni}_{x} \mathrm{Zr}_{100-x}$ amorphous phase, and $E_{\mathrm{Ni}}$ and $E_{\mathrm{Zr}}$ are the lattice energies of the $\mathrm{Ni}$ and $\mathrm{Zr}$ atoms in the ground state, ${ }^{35}$ respectively. The formation energy $\Delta E^{\mathrm{am}}$ for the amorphous phase can be calculated in the MD simulations and is expressed by

$$
\Delta E^{\mathrm{am}}=E_{\mathrm{am}}-\left[x E_{\mathrm{Ni}}+(1-x) E_{\mathrm{Zr}}\right] .
$$


In addition, a $\mathrm{MC}$ simulation was performed to compute the formation energy of the solid solutions, i.e. $\Delta E^{\text {s.s }}$. The $E_{\mathrm{s} . \mathrm{s}}$ is defined as the energy per atom of the $\mathrm{Ni}_{x} \mathrm{Zr}_{100-x}$ solid solutions, and the formation energy $\Delta E^{\text {s.s }}$ of the $\mathrm{Ni}_{x} \mathrm{Zr}_{100-x}$ solid solutions can be calculated by

$$
\Delta E^{\mathrm{s} . \mathrm{s}}=E_{\mathrm{s} . \mathrm{s}}-\left[x E_{\mathrm{Ni}}+(1-x) E_{\mathrm{Zr}}\right]
$$

As a result, the formation energy difference between the amorphous phase and the solid solution can be expressed by

$$
\Delta E^{\mathrm{am}-\mathrm{s} . \mathrm{s}}=E_{\mathrm{am}}-E_{\mathrm{s} . \mathrm{s}}
$$

where $\Delta E^{\text {am-s.s }}$ is defined as the amorphization driving force (ADF). It can be seen from Fig. 1 that both of the formation energies of the amorphous phase and the solid solution phase decrease with the $\mathrm{Zr}$ concentration increasing. However, the ADF first decreases gradually after adding the appropriate $\mathrm{Zr}$ concentration, and after reaching the largest value of $40 \mathrm{at} \% \mathrm{Zr}$, it starts to increase upon further increasing the $\mathrm{Zr}$ concentration. In the present work, three $\mathrm{Ni}_{x} \mathrm{Zr}_{100-x} \mathrm{MGs}$, i.e. $\mathrm{Ni}_{35} \mathrm{Zr}_{65}$, $\mathrm{Ni}_{50} \mathrm{Zr}_{50}$ and $\mathrm{Ni}_{65} \mathrm{Zr}_{35}$, within the glass-forming range of the $\mathrm{Ni}-$ Zr system, were selected as the principle model.

\subsection{Partial pair correlations and structure factor}

At first, the structural changes in the obtained Ni-Zr MGs are monitored using the pair-correlation function (PCF) $g(r)$, which can provide valuable information of liquid and amorphous alloys. The total pair correlation function, $g(r)$, could be obtained using the following formula:

$$
g(r)=\frac{V}{N^{2} \cdot 4 \pi r^{2} \Delta r} \sum_{i}^{N} \sum_{j \neq i}^{N} \delta\left(r-r_{i j}\right),
$$

where $V$ and $N$ are the volume of the calculated cell and the total number of atoms, respectively. The function $\delta(t)$ equals one

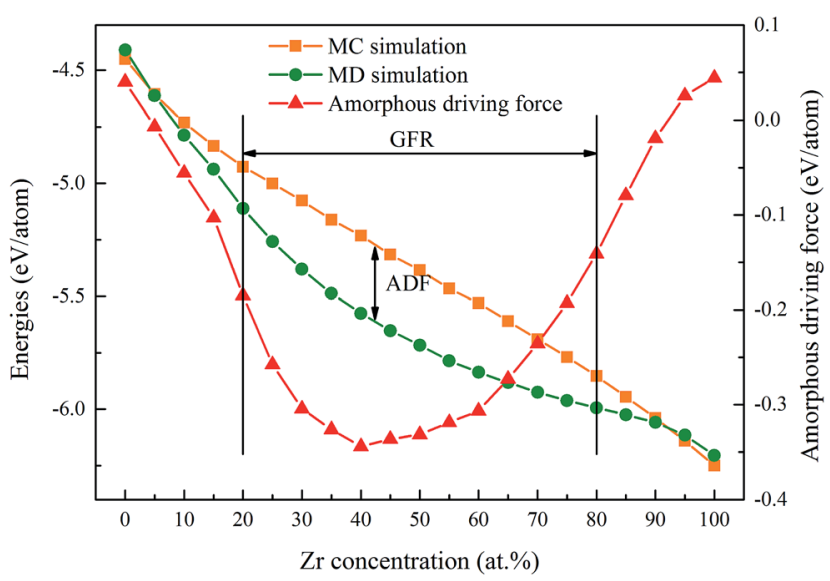

Fig. 1 Formation energies and amorphization driving force of $\mathrm{Ni}-\mathrm{Zr}$ alloys. The glass-forming range of the $\mathrm{Ni}-\mathrm{Zr}$ system is determined to be within 20-80 at\% $\mathrm{Zr}$, and the amorphization driving force is marked by the formation energy difference obtained from the MD and MC simulations.
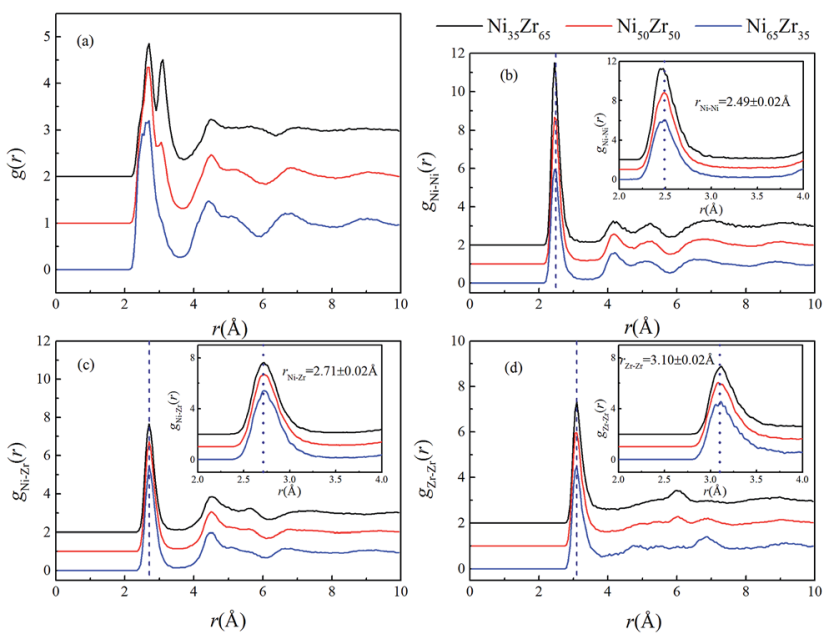

Fig. 2 (a) Total pair-correlation functions (PCFs) and the (b) $\mathrm{Ni}-\mathrm{Ni}$, (c) $\mathrm{Ni}-\mathrm{Zr}$, and (d) $\mathrm{Zr}-\mathrm{Zr}$ partial PCFs of the $\mathrm{Ni}_{35} \mathrm{Zr}_{65}, \mathrm{Ni}_{50} \mathrm{Zr}_{50}$ and $\mathrm{Ni}_{65} \mathrm{Zr}_{35}$ metallic glasses at $300 \mathrm{~K}$. Enlarged partial PCFs in a range of atomic distance 2-4 $\AA$ are also presented.

when $-0.5 \Delta r<t<0.5 \Delta r$ and equals zero otherwise, where $\Delta r$ is the distance interval. Partial PCFs can be obtained by restricting the analysis to the specific elements involved.

Using the atomic coordinates from the MD simulations at $300 \mathrm{~K}$, the total and partial PCFs of the $\mathrm{Ni}_{x} \mathrm{Zr}_{100-x}(x=35,50$ and 65) MGs are displayed in Fig. 2, indicating the amorphous nature of the three alloys. The average interatomic distance $r_{i j}$

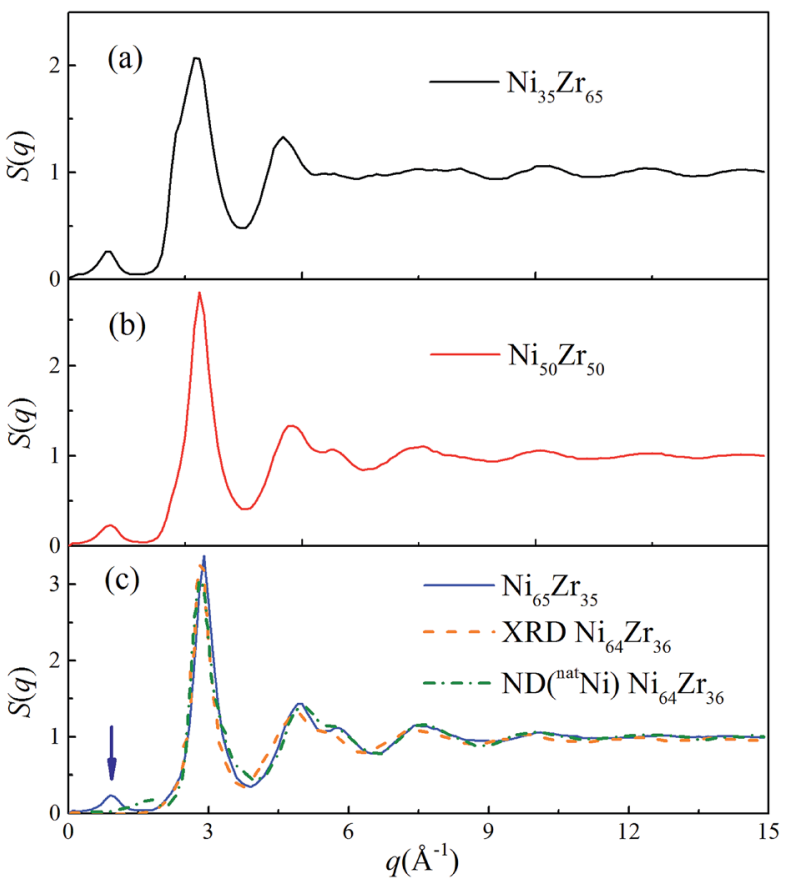

Fig. 3 Total structural factors $S(q)$ for the $\mathrm{Ni}_{35} Z_{r_{65}}, \mathrm{Ni}_{50} \mathrm{Zr}_{50}$ and $\mathrm{Ni}_{65} \mathrm{Zr}_{35}$ metallic glasses at $300 \mathrm{~K}$. The orange and olive dotted lines represent the $S(q)$ for the $\mathrm{Ni}_{64} Z_{r_{36}}$ metallic glasses obtained from the experimental XRD and ND on the ${ }^{n a t} \mathrm{Ni}$-containing samples, ${ }^{28}$ respectively. 
Table 1 Honeycutt-Andersen $(\mathrm{H}-\mathrm{A})$ analysis for the $\mathrm{Ni}_{x} \mathrm{Zr}_{100-x}(x=$ 35,50 and 65) MGs. It should be noted that fcc/hcp indicates fcc- or hcp-like bond pairs of 1421 or 1422, and 142 is used for abbreviation

ico1551 dico1431 dico1541 bcc1661 bcc1441 fcc/hcp142

\begin{tabular}{lllllll}
\hline $\mathrm{Ni}_{35} \mathrm{Zr}_{65}$ & 16.770 & 20.470 & 11.828 & 0.800 & 1.455 & 18.067 \\
$\mathrm{Ni}_{50} \mathrm{Zr}_{50}$ & 25.803 & 21.415 & 12.462 & 1.487 & 1.288 & 12.222 \\
$\mathrm{Ni}_{65} \mathrm{Zr}_{35}$ & 31.917 & 22.475 & 15.341 & 1.847 & 1.242 & 10.890
\end{tabular}

can be determined from the position of the first peak on the $g_{i j}(r)$ function. It can be seen that the $g_{\mathrm{Ni}-\mathrm{Ni}}(r)$ curves of the three alloys overlap around the highest peak with $r=2.49 \pm 0.02 \AA$, while the first peaks of the $g_{\mathrm{Ni}-\mathrm{Zr}}(r)$ and $g_{\mathrm{Zr}-\mathrm{Zr}}(r)$ curves shift to a larger distance of $r=2.71 \pm 0.02 \AA$ and $3.10 \pm 0.02 \AA$, respectively. It follows that the PCF peaks shift to a larger interatomic distance with the enrichment of $\mathrm{Zr}$, considering the fact that $\mathrm{Zr}\left(r_{\mathrm{Zr}}=1.60 \AA\right)$ has a much larger atomic radius than $\mathrm{Ni}\left(r_{\mathrm{Ni}}=1.24 \AA\right) \cdot{ }^{35}$ Similar observations have also been obtained in $\mathrm{Zr}_{2} \mathrm{Ni}$ metallic glass at $300 \mathrm{~K}$ via X-ray diffraction (XRD) experiments and $a b$ initio MD simulations. ${ }^{36}$

In addition, the static structure factor $S(q)$ is a quantity that can be directly measured by scattering experiments such as XRD and neutron diffraction (ND). The $S(q)$ can be recognized as firm evidence to identify the amorphous phase and is calculated by ${ }^{16}$

$$
S(q)=\frac{1}{N}\left\langle\left|\sum_{k=1}^{N} b_{k} \exp \left(i q \cdot r_{k}\right)\right|^{2}\right\rangle,
$$

where $i=\sqrt{-1}, b_{k}$ is the scattering length, and $q$ and $r_{k}$ are the scattering vector and the position vector of atom $k$, respectively. It should be noted that the $S(q)$ calculated by eqn (8) is not normalized.

As shown in Fig. 3, all of the peaks beyond the second peak for the $S(q)$ curves of the $\mathrm{Ni}_{x} \mathrm{Zr}_{100-x}$ MGs have smeared out, exhibiting a typical short-range ordered and long-range disordered feature. The prepeak for the $S(q)$ curves in the reciprocal space marked by the arrow should correspond to a certain medium-range correlation in real space. ${ }^{37}$ For comparison, the $S(q)$ curve of the $\mathrm{Ni}_{64} \mathrm{Zr}_{36}$ MGs obtained from the experimental XRD and ND for the ${ }^{\text {nat }} \mathrm{Ni}$-containing samples are also shown in
Fig. $3 .^{28}$ Although some compositional differences exist, the simulated $S(q)$ in the present study exhibits an acceptable match with the experimental ones regarding the height of the peaks and the overall shape of the curve, confirming the reliability of the atomistic structures generated from the solid-state amorphization.

\subsection{Local atomic structure analysis}

In the present work, 3D atomic models were used to analyze the local atomic configuration. Here we used the HoneycuttAndersen pair analysis, cluster alignment method, coordination number and Voronoi tessellation method to analyze the local structural order in the simulated Ni-Zr MG model.

To provide a microscopic picture of the structural origin for the $\mathrm{Ni}_{x} \mathrm{Zr}_{100-x} \mathrm{MGs}$, Honeycutt-Andersen pair analysis was first applied to characterize the topological short-range order (SRO). With the Ni concentration increasing, one can see from Table 1 that the fraction of the bond pairs with 1441 and 142 indices decreases, whereas the fraction of the bond pairs with 1551, 1541, 1431 and 1661 indices increases. Meanwhile, a large number of the icosahedral ordering 1551 index are found in the $\mathrm{Ni}_{x} \mathrm{Zr}_{100-x}$ MGs, suggesting that the local fivefold symmetry is indeed encouraged in metallic glasses. Besides, the distorted fivefold bond pairs with 1431 and 1541 indices are dominant in these metallic glasses, covering a fraction of $32-38 \%$. Moreover, the fcc- and hcp-like pairs with 1421 and 1422 indices cover a relatively larger fraction due to the original crystal structure, while only a small fraction of the bcc-like pairs with 1661 and 1441 indices are discovered in the $\mathrm{Ni}_{x} \mathrm{Zr}_{100-x}$ MGs. It has been reported that the fcc or hep arrangements are shown to minimize the system energy, because of the smaller binding energies than that of the icosahedral order. ${ }^{38}$ Therefore, it is revealed that the local structures in the $\mathrm{Ni}_{x} \mathrm{Zr}_{100-x}$ MGs exhibit a combination of icosahedral-, fcc- and hep-like configurations.

To give more information, the local structure was quantitatively analyzed using a cluster alignment method. The distribution of the alignment score for the $\mathrm{Ni}_{x} \mathrm{Zr}_{100-x}(x=35,50$ and 65) MGs is plotted in Fig. 4. The smaller the alignment score, the less deviation the aligned cluster has compared with the template. In this case, all of the MG samples exhibit better (a)

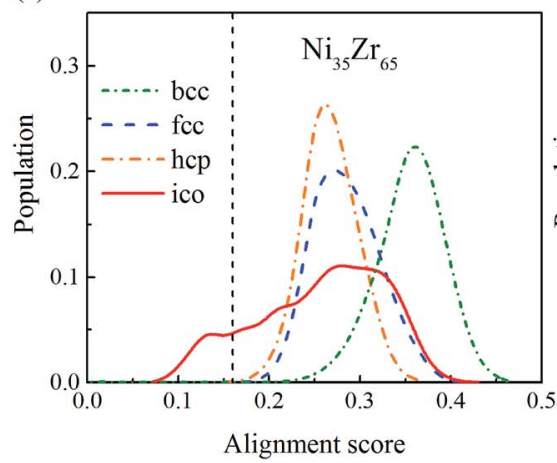

(b)

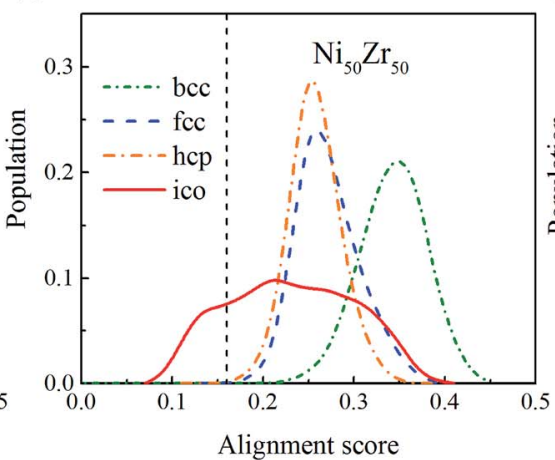

(c)

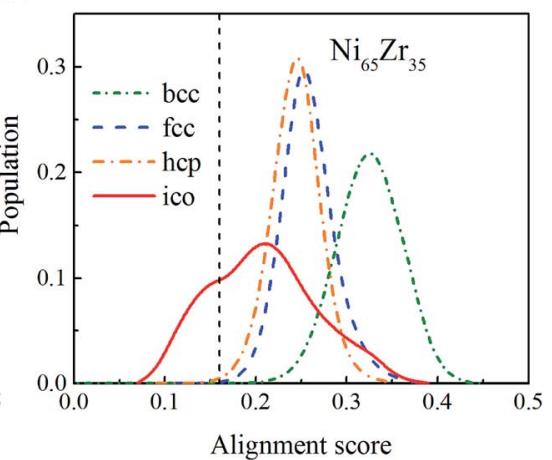

Fig. 4 The distribution of the alignment score for the Ni-centered clusters extracted from the (a) $\mathrm{Ni}_{35} \mathrm{Zr}_{65}$, (b) $\mathrm{Ni}_{50} \mathrm{Zr}_{50}$ and (c) $\mathrm{Ni}_{65} \mathrm{Zr}_{35}$ metallic glasses. 

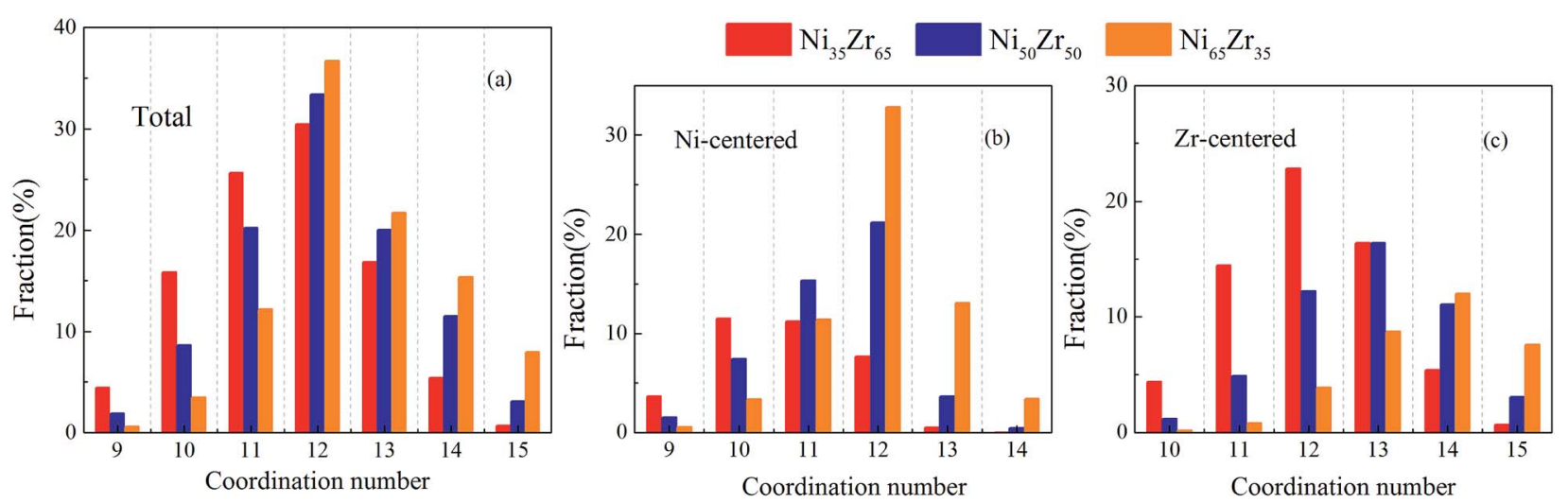

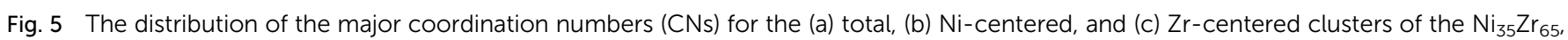
$\mathrm{Ni}_{50} \mathrm{Zr}_{50}$ and $\mathrm{Ni}_{65} \mathrm{Zr}_{35}$ metallic glasses.

icosahedral order when compared with the bcc-, fcc- and heplike configurations, as the distribution function gradually shifts towards the lower values of the alignment score.

In addition to the cluster alignment method and HoneycuttAndersen pair analysis, the coordination number and Voronoi tessellation method are also widely used to provide a more complete geometrical construction of the central atom in relation to the neighboring atoms. Specifically, an envelope of a family of perpendicular bisectors between the central atom and all of the neighboring atoms constitutes the surface of a Voronoi polyhedron for the central atom, indexed by $\left\langle n_{3}, n_{4}\right.$, $\left.n_{5}, n_{6}, \ldots\right\rangle$, where $n_{i}$ denotes the number of $i$-edged faces of the Voronoi polyhedron and $\Sigma n_{i}$ is the total $\mathrm{CN}$.

We present the overall CN distributions for the $\mathrm{Ni}_{x} \mathrm{Zr}_{100-x}$ $(x=35,50$ and 65) MGs in Fig. 5(a). It can be seen that the dominant clusters for these MGs are centered around $\mathrm{CN}=12$. As the Ni content increases, the total CN distributions in the MGs have undergone a noticeable decrease in the fraction of low-CN clusters, whereas an inverse trend is observed for high$\mathrm{CN}$ clusters. This is mainly attributed to the fact that an increasing percentage of smaller $\mathrm{Ni}$ atoms are supposed to serve as solutes, which therefore favors higher CNs. To further investigate the local packing around each individual type of atom, the evolution of the $\mathrm{CN}$ distributions around the $\mathrm{Ni}$ and $\mathrm{Zr}$ atoms was analyzed and is shown in Fig. 5(b) and (c). It is revealed that the CNs of the Ni-centered clusters are centered around $\mathrm{CN}=11$ and 12 , while the $\mathrm{CNs}$ of the $\mathrm{Zr}$-centered clusters are centered around high-CN clusters. For the $\mathrm{Ni}_{65} \mathrm{Zr}_{35} \mathrm{MGs}$, the $\mathrm{Ni}$ atoms with dominant concentration are more likely to serve as solvents and also act as 'glue' for the glassy structure. ${ }^{39}$ Therefore, it is plausible to expect that the $\mathrm{Zr}$ atoms could more readily adopt a favored local packing at their preference, while the $\mathrm{Ni}$ atoms would experience more flexible local environments.

The populations of the dominant Voronoi polyhedra around the constituent elements for the $\mathrm{Ni}_{x} \mathrm{Zr}_{100-x}(x=35,50$ and 65) MGs were then calculated and are illustrated in Fig. 6(a), (b) and (c), respectively. One can see that the dominant polyhedra in the $\mathrm{Ni}_{x} \mathrm{Zr}_{100-x}(x=35,50$ and 65) MGs are $\langle 0,0,12,0\rangle,\langle 0,2,8,1\rangle,\langle 0$, $2,8,2\rangle$ and $\langle 0,1,10,2\rangle$. It is well-known that the icosahedron $\langle 0$, $0,12,0\rangle$ has been widely considered as being responsible for reducing the total energy and stabilizing the glassy state. ${ }^{36}$ In
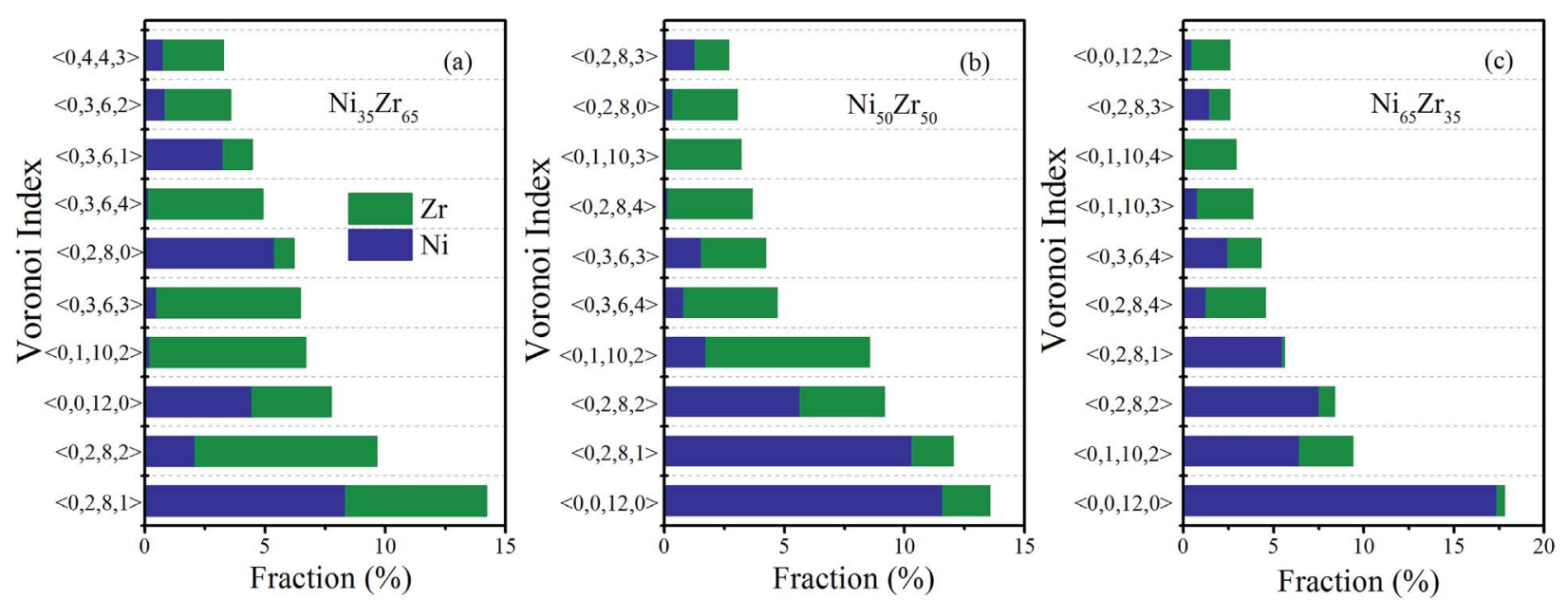

Fig. 6 Fraction of the dominant Voronoi clusters centered on $\mathrm{Ni}$ and $\mathrm{Zr}$ atoms for the (a) $\mathrm{Ni}_{35} \mathrm{Zr}_{65}$, (b) $\mathrm{Ni}_{50} \mathrm{Zr}_{50}$ and (c) $\mathrm{Ni}_{65} \mathrm{Zr}_{35}$ metallic glasses. 
(a)

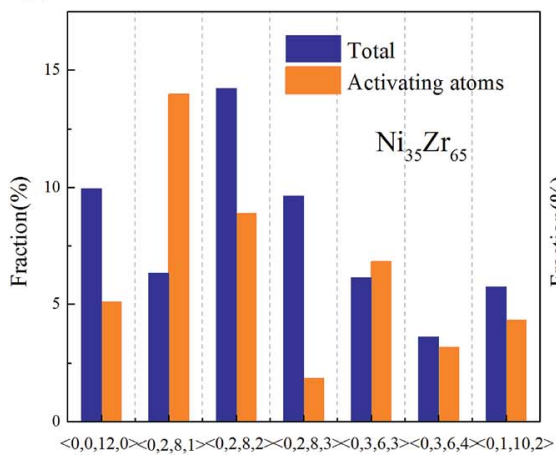

(b)

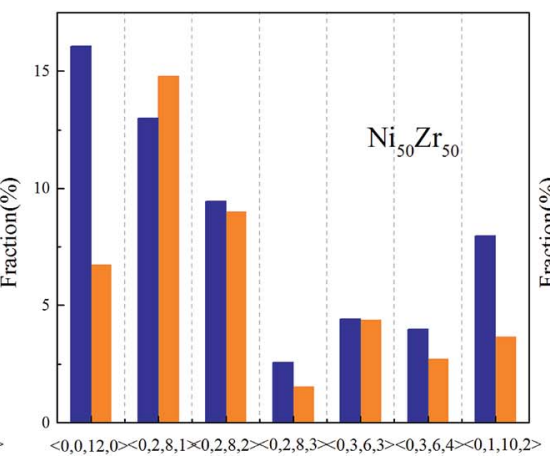

(c)

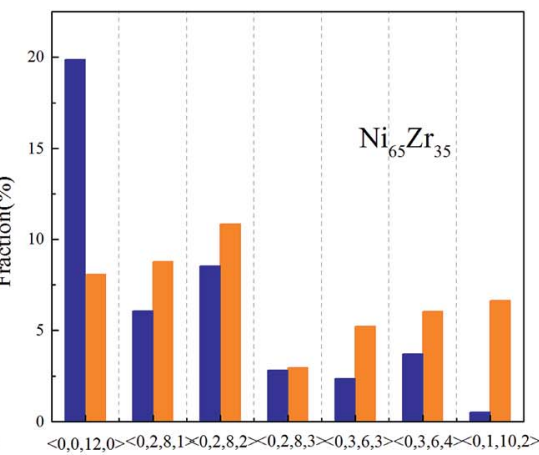

Fig. 7 Fraction of the dominant Voronoi clusters centered on the $\mathrm{Ni}$ and $\mathrm{Zr}$ atoms for the (a) $\mathrm{Ni}_{35} \mathrm{Zr}_{65}$, (b) $\mathrm{Ni}_{50} \mathrm{Zr}_{50}$ and (c) Ni $\mathrm{Ni}_{65} \mathrm{Zr} 35$ metallic glasses.

addition, the distorted icosahedra $\langle 0,2,8,1\rangle,\langle 0,2,8,2\rangle$ and $\langle 0$, $1,10,2\rangle$ are also populated around $\mathrm{Ni}$ and $\mathrm{Zr}$ due to the fact that these clusters have 11-13 neighboring atoms and a high percentage of fivefold-coordinated vertices, thus making them similar to icosahedra. The above analysis results match well with the cluster alignment method and Honeycutt-Andersen pair analysis, indicating that the icosahedra or distorted icosahedra have a rather high fraction in the $\mathrm{Ni}_{x} \mathrm{Zr}_{100-x}(x=35,50$ and 65) MGs.

\subsection{Internal friction structure features}

According to the cooperative shear model (CSM), ${ }^{\mathbf{4 0 , 4 1}}$ the atoms that jump more than half of the distance to the average nearest neighbor govern the internal friction in the model MGs, and are defined as activating atoms in the present work. As shown in Fig. 7, the lower-CN clusters, such as $\langle 0,2,8,1\rangle$, occupy more of the population in the activating atoms and may play a significant role in the atomic mobility. In order to further illustrate the relationship between the activating atoms and the local atomic structure, the perspective view and corresponding cluster alignment score of the activating atoms in the $\mathrm{Ni}_{x} \mathrm{Zr}_{100-x}(x=$ 35,50 and 65) MGs at $900 \mathrm{~K}$ and $f_{\omega}=1 \mathrm{GHz}$ after 20 cycle deformations are presented in Fig. 8. It is found that the lower the Ni concentration, the higher the concentration of the activating atoms, and vice versa. This may be understood in the view of the coordination distribution. It can be seen from Fig. 5 that the $\mathrm{Ni}_{65} \mathrm{Zr}_{35}$ MGs prefer the lower-CN clusters, which are prone to move faster with a smaller size and number of atoms. (a)

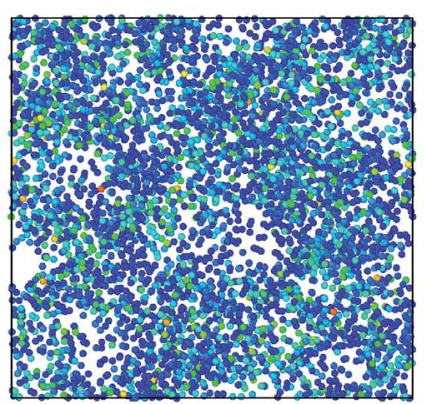

(d)

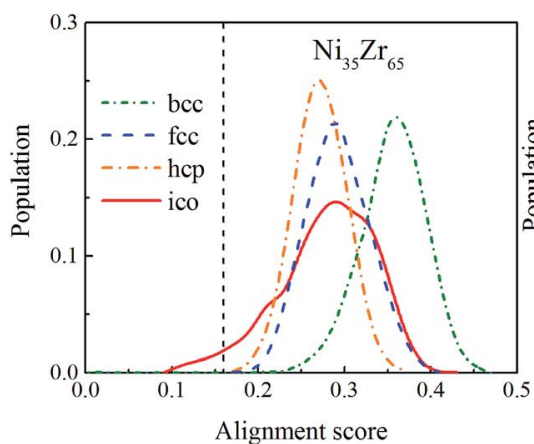

(b)

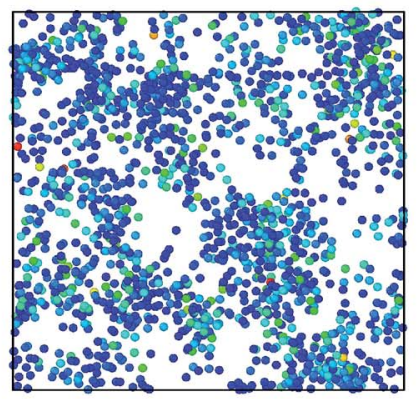

(e)

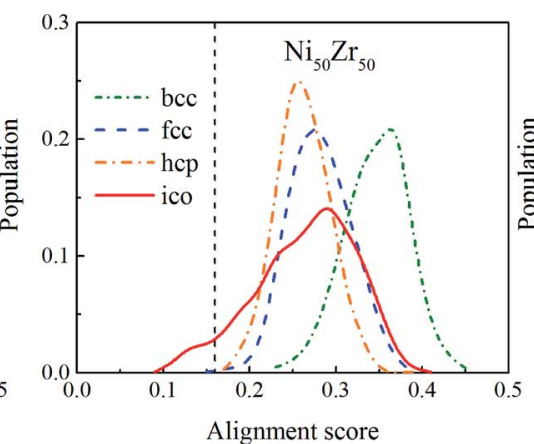

(c)

(f)
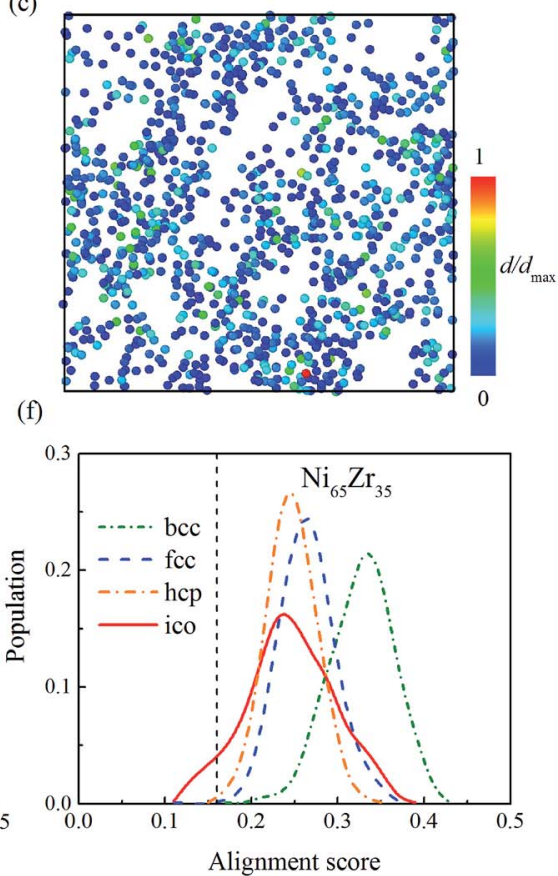

Fig. 8 The perspective view and corresponding cluster alignment score of the activating atoms at $900 \mathrm{~K}$ and $f_{\omega}=1 \mathrm{GHz}$ after 20 period cycles in the $\mathrm{Ni}_{x} \mathrm{Zr}_{100-x}(x=35,50$ and 65) MGs, aligned from left to right, and the atoms are colored by the displacement $d$ from the original position. 


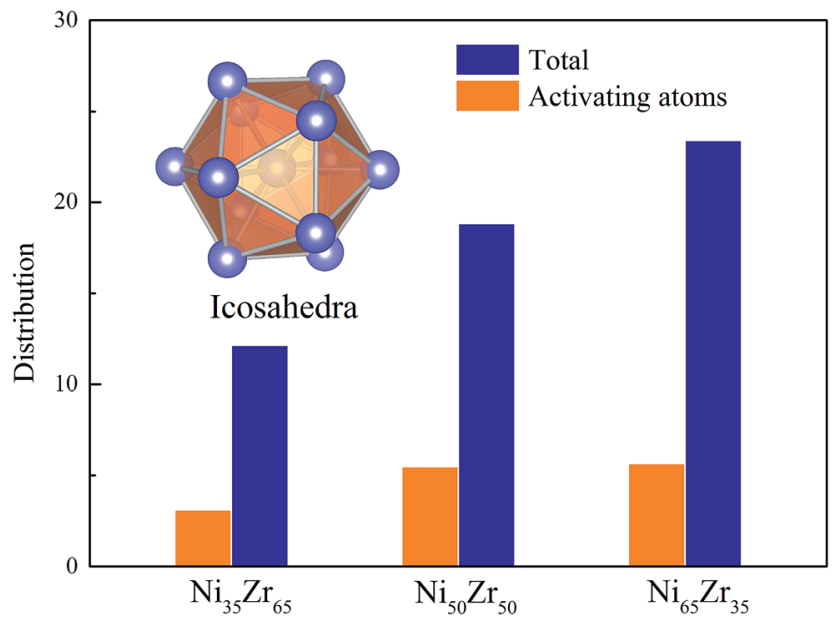

Fig. 9 The fraction of icosahedral clusters for all atoms and for the activating atoms in the $\mathrm{Ni}_{x} \mathrm{Zr}_{100-x}(x=35,50$ and 65) MGs. A cutoff alignment score of 0.16 was used to collect the icosahedra.

Compared with the bcc, fcc and hcp configurations, the activating atoms prefer the icosahedra order due to the lower value of the cluster alignment score as shown in Fig. 8(d)-(f). A cutoff alignment score of $0.15-0.16$, where the population is close to the first peak of the icosahedral order distribution for each alloy in Fig. 4, was applied to collect icosahedral clusters for all the samples. Furthermore, the population of the icosahedral clusters for all atoms and activating atoms collected from $\mathrm{Ni}_{x} \mathrm{Zr}_{100-x}$ $(x=35,50$ and 65) MGs are displayed in Fig. 9. By comparing the fraction of icosahedral clusters for all atoms and the activating atoms, there exists a large difference in the fraction for both groups, indicating that the icosahedron is less sensitive to the faster mobility. However, whether there exists the determined local atomic structure influencing the atomic mobility still needs further insightful discussion.

\section{Conclusions}

Taking a long-range empirical $\mathrm{Ni}-\mathrm{Zr}$ n-body potential as the starting base, Molecular Dynamics and Monte Carlo simulations not only pinpoint the glass forming range of the $\mathrm{Ni}-\mathrm{Zr}$ system as $20-80$ at\% $\mathrm{Zr}$, but also predict the amorphization driving force with the peak value of 40 at\% $\mathrm{Zr}$. The paircorrelation function $g(r)$ and static structure factor $S(q)$ curves of the $\mathrm{Ni}_{x} \mathrm{Zr}_{100-x}(x=35,50$ and 65) alloys present a typical short-range ordered and long-range disordered feature. Furthermore, the obtained atomic structures have been characterized by the pair-correlation functions, static structure factor, Honeycutt-Andersen pair analysis, coordination number, Voronoi tessellation method and cluster alignment method. It is revealed that the $\mathrm{Ni}_{x} \mathrm{Zr}_{100-x}(x=35,50$ and 65) MGs exhibit a combination of the icosahedral-, fcc- and hcp-like configurations, while the icosahedra or distorted icosahedra cover a dominant fraction. Interestingly, the lower-CN clusters, such as $\langle 0,2,8,1\rangle$, occupy more of the population in the activating atoms and may play a significant role in the atomic mobility. By comparing the fraction of icosahedral clusters for all atoms and for the activating atoms, there exists a large difference in the fraction for both groups, indicating that the icosahedron is less sensitive to the faster mobility.

\section{Acknowledgements}

The authors are grateful for the financial support from the National Natural Science Foundation of China (51571129, 51631005), the Administration of Tsinghua University, and Tsinghua Fudaoyuan Research Fund.

\section{References}

1 M. D. Ediger and P. Harrowell, J. Chem. Phys., 2012, 137, 080901.

2 S. Adibi, P. S. Branicio and R. Ballarini, RSC Adv., 2016, 6, 13548-13553.

3 J. C. Zhang, C. Chen, Q. X. Pei, Q. Wan, W. X. Zhanga and Z. D. Sha, RSC Adv., 2016, 6, 100899-100907.

4 H.-L. Chen, C.-H. Su, S.-P. Ju, S.-H. Liu and H.-T. Chen, RSC Adv., 2015, 5, 103925-103935.

5 J. Varghese, T. Siponkoski, M. Teirikangas, M. T. Sebastian, A. Uusimäki and H. Jantunen, ACS Sustainable Chem. Eng., 2016, 4, 3897-3904.

6 J. Varghese, S. Gopinath and M. T. Sebastian, Mater. Chem. Phys., 2013, 137, 811-815.

7 N. V. Priezjev, Phys. Rev. E: Stat., Nonlinear, Soft Matter Phys, 2013, 87, 052302.

8 K. Martens, L. Bocquet and J.-L. Barrat, Phys. Rev. Lett., 2011, 106, 156001.

9 A. Lemaître and C. Caroli, Phys. Rev. Lett., 2009, 103, 065501. 10 N. P. Bailey, J. Schiøtz, A. Lemaître and K. W. Jacobsen, Phys. Rev. Lett., 2007, 98, 095501.

11 E. Lerner and I. Procaccia, Phys. Rev. E: Stat., Nonlinear, Soft Matter Phys., 2009, 79, 066109.

12 C. C. Wang, Y. W. Mao, Z. W. Shan, M. Dao, J. Li, J. Sun, E. Ma and S. Suresh, Proc. Natl. Acad. Sci. U. S. A., 2013, 110, 19725-19730.

13 G. Sainath, P. Rohith and B. K. Choudhary, Trans. Indian Inst. Met., 2016, 69, 489-493.

14 Z. W. Wu, M. Z. Li, W. H. Wang and K. X. Liu, Phys. Rev. B: Condens. Matter Mater. Phys., 2013, 88, 054202.

15 Y. Q. Cheng, H. W. Sheng and E. Ma, Phys. Rev. B: Condens. Matter Mater. Phys., 2008, 78, 014207.

16 C. Suryanarayana and M. G. Norton, X-ray diffraction: a practical approach, Plenum Press, New York, 1998.

17 J. D. Honeycutt and H. C. Andersen, J. Phys. Chem., 1987, 91(19), 4950-4963.

18 J. L. Finney, Proc. R. Soc. London, Ser. A, 1970, 319, 479-493. 19 Y. Sun, F. Zhang, Z. Ye, Y. Zhang, X. W. Fang, Z. J. Ding, C. Z. Wang, M. I. Mendelev, R. T. Ott, M. J. Kramer and K. M. Ho, Sci. Rep., 2016, 6, 23734.

20 M. H. Yang, J. H. Li and B. X. Liu, Sci. Rep., 2016, 6, 29722. 21 Y. Dai, J. H. Li and B. Liu, J. Appl. Phys., 2011, 109, 053505. 22 J. H. Li, S. Z. Zhao, Y. Dai, Y. Y. Cui and B. X. Liu, J. Appl. Phys., 2011, 109, 113538. 
23 H. B. Yu, K. Samwer, W. H. Wang and H. Y. Bai, Nat. Commun., 2013, 4, 2204.

24 H. B. Yu, R. Richert, R. Maaß and K. Samwer, Phys. Rev. Lett., 2015, 115, 135701.

25 X. W. Fang, C. Z. Wang, Y. X. Yao, Z. J. Ding and K. M. Ho, Phys. Rev. B: Condens. Matter Mater. Phys., 2010, 82, 184204.

26 X. W. Fang, L. Huang, C. Z. Wang, K. M. Ho and Z. J. Ding, J. Appl. Phys., 2014, 115, 053522.

27 Y. Sun, Y. Zhang, F. Zhang, Z. Ye, Z. J. Ding, C. Z. Wang and K. M. Ho, J. Appl. Phys., 2016, 120, 015901.

28 I. Kabana, P. Jóváric, V. Kokotind, O. Shuleshovaa, B. Beuneue, K. Sakslf, N. Matterna, J. Eckerta and A. Greerg, Acta Mater., 2013, 61, 2509-2520.

29 M. H. Yang, J. H. Li and B. X. Liu, RSC Adv., 2015, 5, 16400.

30 A. Inoue, T. Zhang and T. Masumoto, Mater. Trans., JIM, 1990, 31, 177-183.

31 Z. P. Lu and C. T. Liu, Acta Mater., 2002, 50, 3501-3512.
32 H. Li, X. D. Dai, S. H. Liang, K. P. Tai, Y. Kong and B. X. Liu, Phys. Rep., 2008, 455, 1-134.

33 M. H. Yang, S. N. Li, Y. Li, J. H. Li and B. X. Liu, Phys. Chem. Chem. Phys., 2015, 17, 13355-13365.

34 A. W. Weeber and H. Bakker, J. Phys. F: Met. Phys., 1988, 18, 1359.

35 C. Kittel, Introduction to solid state physics, Wiley, New York, 7th edn, 1996.

36 L. Huang, C. Z. Wang, S. G. Hao, M. J. Kramer and K. M. Ho, Phys. Rev. B: Condens. Matter Mater. Phys., 2010, 81, 094118.

37 Y. Q. Cheng and E. Ma, Prog. Mater. Sci., 2011, 56, 379-473.

38 F. C. Frank, Proc. R. Soc. London, Ser. A, 1952, 215, 43-46.

39 H. W. Sheng, W. K. Luo, F. M. Alamgir, J. M. Bai and E. Ma, Nature, 2006, 439, 419-425.

40 W. L. Johnson and K. Samwer, Phys. Rev. Lett., 2005, 95, 195501.

41 H. B. Yu and K. Samwer, Phys. Rev. B: Condens. Matter Mater. Phys., 2014, 90, 144201. 